\title{
LEARNING-BASED SCAN PLANE IDENTIFICATION FROM FETAL HEAD ULTRASOUND IMAGES*
}

\author{
Xiaoming Liu ${ }^{a}$, Pavan Annangi ${ }^{b}$, Mithun Gupta ${ }^{b}$, Bing Yu ${ }^{a}$, Dirk Padfield ${ }^{a}$, Jyotirmoy \\ Banerjee $^{b}$, Kajoli Krishnan ${ }^{b}$ \\ ${ }^{a}$ GE Global Research, One Research Circle, Niskayuna, NY 12309, USA \\ $\{$ liux, yu,padfield\}@research.ge.com \\ ${ }^{b}$ GE Global Research, Phase 2, Hoodi Village, Whitefield Road, Bangalore, India \\ \{pavan.annangi, mithun.dasgupta, jyotirmoy.banerjee,kajoli.krishnan\}@ge.com
}

\begin{abstract}
Acquisition of a clinically acceptable scan plane is a pre-requisite for ultrasonic measurement of anatomical features from B-mode images. In obstetric ultrasound, measurement of gestational age predictors, such as biparietal diameter and head circumference, is performed at the level of the thalami and cavum septum pelucidi. In an accurate scan plane, the head can be modeled as an ellipse, the thalami looks like a butterfly, the cavum appears like an empty box and the falx is a straight line along the major axis of a symmetric ellipse inclined either parallel to or at small angles to the probe surface. Arriving at the correct probe placement on the mother's belly to obtain an accurate scan plane is a task of considerable challenge especially for a new user of ultrasound. In this work, we present a novel automated learning-based algorithm to identify an acceptable fetal head scan plane. We divide the problem into cranium detection and a template matching to capture the composite "butterfly" structure present inside the head, which mimics the visual cues used by an expert. The algorithm uses the stateof-the-art Active Appearance Models techniques from the image processing and computer vision literature and tie them to presence or absence of the inclusions within the head to automatically compute a score to represent the goodness of a scan plane. This automated technique can be potentially used to train and aid new users of ultrasound.
\end{abstract}

Keywords: Ultrasound, biparietal diameter, head circumference, scan plane, thalami, falx, cavum, active appearance model, fetal head

\section{DESCRIPTION OF THE PURPOSE}

One and two-dimensional measurements such as those of thickness, perimeter and area are a part of routine ultrasound examination in cardiac, obstetric, abdominal and vascular imaging. In two-dimensional (2D) ultrasound, acquisition of B-mode images in a clinically acceptable scan plane is an important first step prior to manually placing the caliper to make a measurement. With extensive training and practice, expert sonographers can typically acquire the scan plane of interest within tens of seconds. As ultrasound devices shrink in size and cost, their reach extends to novice users at the point-of-care who could benefit from applications that simplify and automate the clinical workflow. ${ }^{1}$

Two-dimensional ultrasound is routinely used in obstetric applications to monitor fetal development. Ultrasound measurements of specific features of fetal anatomy such as the head, abdomen, and femur are used in the determination of gestational age (GA), assessment of growth patterns, and identification of anomalies. In order to get accurate and reproducible gestational age estimates, each of these image acquisitions are to be made in some predefined protocols. In common clinical practice, the ultrasound transducer is moved over the maternal abdomen until the fetal head is visible in a standard scan plane shown in Figure 1. In this scan plane, the user looks for the midline falx, cavum septum pelucidi, and paired thalami which form a "butterfly" structure. There is no fixed one-to-one correlation between external landmarks and probe movement with internal anatomical features since the fetus can be oriented in different directions in the uterine cavity. Maternal habits vary and so

Further author information: (Send correspondence to Xiaoming Liu, E-mail: liux@research.ge.com) 
$\rightarrow$ Midline falx (Third ventricle)

$\rightarrow$ Paired thalami

Cavum Septum Pellucidum

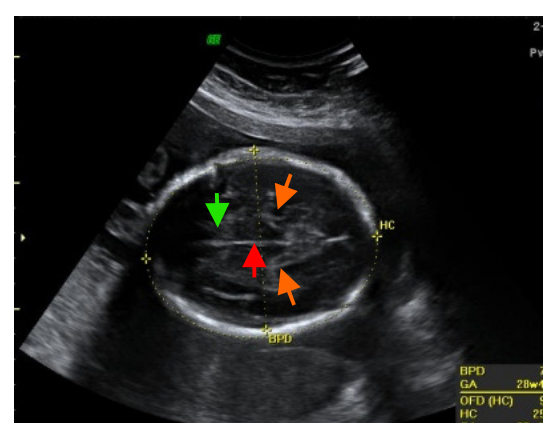

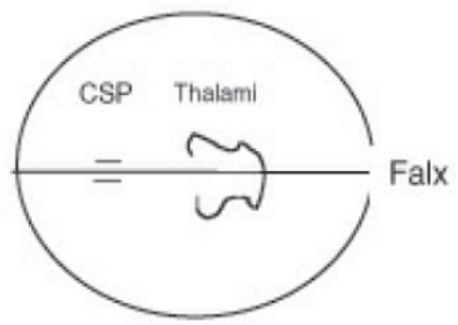

Correct head placement for BPD and $\mathrm{HC}$ measurement

Figure 1. Anatomical structures in a correct fetal head scan image.

does the size of the fetus and its presentation in an ultrasound image. As a result, the image acquisition of the correct scan plane is both a challenging and time consuming task for non-expert users. The current methodology aims at assisting novice users of ultrasound by providing an automated feedback on the quality of scan plane during the scanning procedure.

As learned from the experienced sonographers, the key factor to claim a correct scan plane is the presence of the paired thalami structure. Hence, following the same principle, we will utilize automatic machine learning algorithms to detect this structure after the head region is localized. In the computer vision community, there have been extensive prior efforts on detecting an object instance with variability in both its shape and appearance. In particular, learning-based approaches, the Active Appearance Models (AAM) ${ }^{2-5}$ and its variations such as Boosted Appearance Models ${ }^{6,7}$ and Boosted Ranking Models, ${ }^{8,9}$ are popular approaches since the variations of the object class (or the structure) can be explicitly explored based on a set of representative training samples. Hence, we choose to employ an advanced AAM approach to find such structures in the images. Furthermore, we also use a score function to calculate the correctness of head scan plane based on the detected structure. We test our approach with a set of 94 still frames of fetal head images, each with an expert label of whether it is a correct scan plane.

\section{METHOD AND ALGORITHM}

We solve the scan plane identification problem using a three-step method, as shown in Figure 2. Given an ultrasound scan image, we first attempt to detect the fetal head by the method of Banerjee and Krishnan.$^{10}$ If a head is detected, we rectify the head region based on the estimated head orientation. It is well known that the presence of a composite structure, such as the butterfly-shaped thalami and the falx, is a strong indication of correct scan planes. Hence, the second step is to detect whether this structure exists in the center part of the head region. Due to large amount of variations in the appearance and shape of this structure, we employ Active Appearance Models (AAM) for the task of structure fitting. Finally, we utilize a learning-based score function to calculate the correctness of the head scan plane given the detected structure.

\subsection{Structure Fitting}

The shape model and appearance model part of an AAM are trained with a representative set of images. The distribution of landmarks is modeled as the shape model. Given $M$ ultrasound images with good scan planes, each image $I_{m}$ is manually labeled with a set of $v$ landmarks, whose collection is treated as one observation for the shape model, $\mathbf{s}=\left[x_{1}, y_{1}, x_{2}, y_{2}, \ldots, x_{v}, y_{v}\right]^{\mathrm{T}}$. Finally eigen analysis is applied on the observations set and the resultant linear shape space can represent any shape as: $\mathbf{s}(\mathbf{P})=\mathbf{s}_{0}+\sum_{i=1}^{n} p_{i} \mathbf{s}_{i}$, where $\mathbf{s}_{0}$ is the mean shape, $\mathbf{s}_{i}$ is the $i^{\text {th }}$ shape basis, and $\mathbf{p}=\left[p_{1}, p_{2}, \ldots, p_{n}\right]$ is the shape coefficient. With the shape basis, a mapping function from the model coordinate system to the observation coordinate system can be defined as $\mathbf{W}(\mathbf{x} ; \mathbf{p})$, where $\mathbf{x}$ is a pixel coordinate defined by the mean shape $\mathbf{s}_{0}$.

After the shape model is trained, the appearance model is obtained as follows. Each image is warped into the mean shape based on the piece-wise affine transformation between its shape instance and the mean shape. 


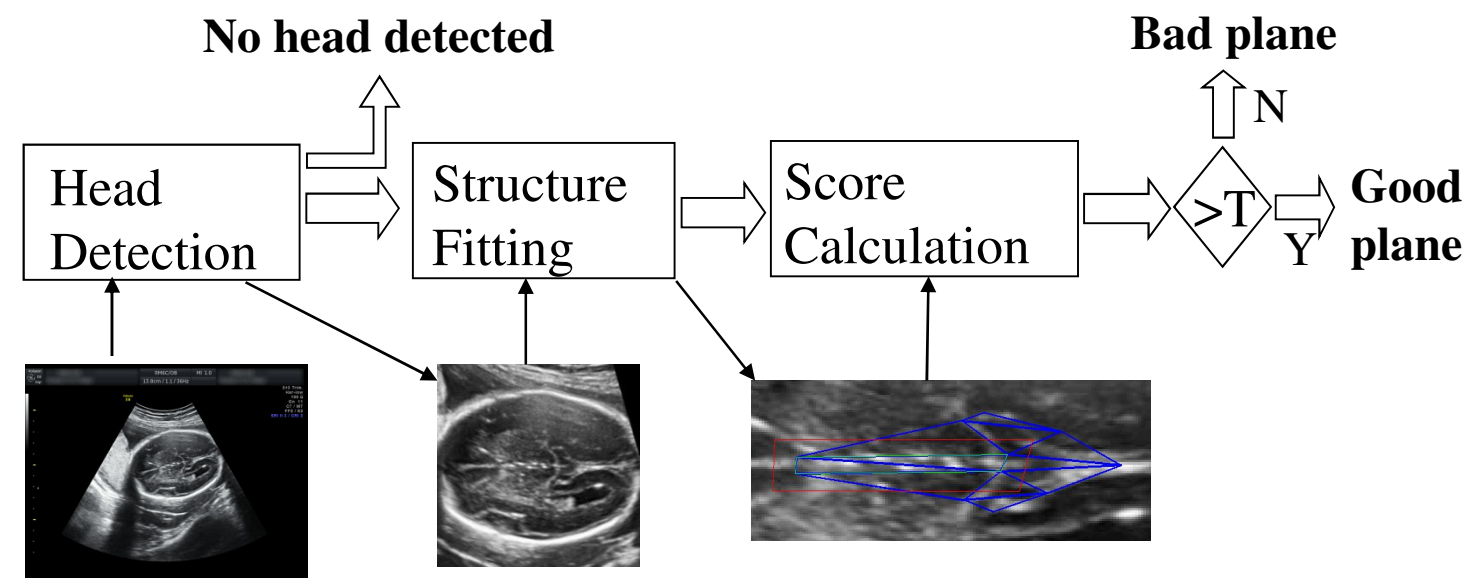

Figure 2. The overview of our 3-step approach to scan plane identification.

These shape-normalized appearances from all training images are fed into eigen analysis and the resultant model can represent any appearance as: $A(\mathbf{x} ; \lambda)=A_{0}(\mathbf{x})+\sum_{i=1}^{m} \lambda_{i} A_{i}(\mathbf{x})$, where $A_{0}$ is the mean appearance, $A_{i}$ is the $i^{t h}$ appearance basis, and $\lambda=\left[\lambda_{1}, \lambda_{2}, \ldots, \lambda_{m}\right]$ is the appearance coefficient. Figure 3 shows the AAM trained using 51 ultrasound images.

From the modeling side, the AAM generated above can synthesize the butterfly structure with arbitrary shape and appearance within a certain population. On the other hand, model fitting is used by AAM to explain a good scan plane image by finding the optimal shape and appearance coefficients such that the synthesized image is as close to the observation image as possible. This leads to the cost function used in model fitting, ${ }^{2}$ $J(\mathbf{p}, \lambda)=\sum_{\mathbf{x} \in \mathbf{s}_{0}}[I(\mathbf{W}(\mathbf{x} ; \mathbf{p}))-A(\mathbf{x} ; \lambda)]^{2}$, which is the mean-square-error (MSE) between the image warped from the observation $I(\mathbf{W}(\mathbf{x} ; \mathbf{p}))$ and the synthesized appearance model instance $A(\mathbf{x} ; \lambda)$.

Traditionally this minimization of the aforementioned cost function is solved by gradient decent methods. Baker and Matthews ${ }^{11}$ proposed the Inverse Compositional and Simultaneously Inverse Compositional methods that greatly improve the fitting performance. Their basic idea is that the role of appearance templates and the input image is switched when computing $\Delta \mathbf{P}$. Thus a large portion of time-consuming steps in parameter estimation can be pre-computed and remain constant during the fitting iteration.

In our application, applying the AAM to structure fitting problem means that AAM attempts to optimize the shape/appearance coefficients initialized by the mean shape $\mathbf{s}_{0}$ at the center of the rectified head region. To improve the fitting performance, we have applied a number of enhancements to this approach, among which model enhancement and video-based fitting are the major ones. Firstly, we employ the iterative model enhancement technique $^{3}$ in learning an AAM. The basic idea is that by iteratively performing model learning and image
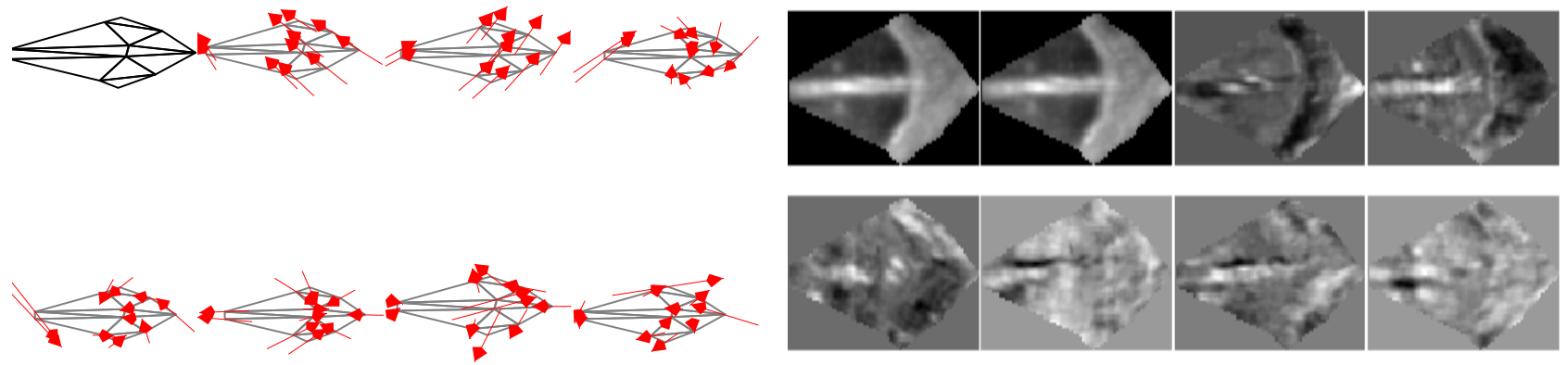

Figure 3. The mean and top 7 eigenvectors of the shape model (left) and the appearance model (right), which represents the possible shape and appearance variations of the "butterfly" structure. 
alignment, the manually labeled landmarks can be modified such that an enhanced model can be generated. This enhanced model not only improves the fitting performance, but also increases its efficiency. Secondly, conventional AAM-based fitting rarely takes advantage of the temporal correlation while applying to ultrasound scan sequences. We employ the video-based approach ${ }^{5}$ that can automatically make use of the fitting results in previous scan frames, in addition to the generically trained AAM model. This is particularly useful for the fitting to a continuous sequence of scan frames.

\subsection{Score Function}

After AAM fitting converges, one straightforward way is to use the computed $J(\mathbf{p}, \lambda)$ as the correctness measurement for a scan plane. However, it has an obvious drawback. Although we wish that $J(\mathbf{p}, \lambda)$ can fully indicate the appearance similarity between the test frame and the model, the fitting inaccuracy of the shape will also contribute to the final appearance residue $J(\mathbf{p}, \lambda)$. Hence, we propose to learn a classifier and use that as the score function. We select a set of positive samples, which are the correct scan plane images with AAM-estimated landmarks, and a set of negative samples, which are the incorrect scan plane images with AAM-estimated landmarks. The feature representation for both classes is the warped image pixels within the mean shape space, $I(\mathbf{W}(\mathbf{x} ; \mathbf{p}))$. Linear discriminative analysis $(\mathrm{LDA})^{12}$ is utilized to learn a classifier for the given samples. Since we only have two classes, the LDA classifier is a one-dimensional vector acting as the weights for each pixel in the feature space $I(\mathbf{W}(\mathbf{x} ; \mathbf{p}))$.

\section{EXPERIMENTAL RESULTS}

The test data set consists of 94 fetal head scan images. These images were subjectively rated by an expert clinician as correct/incorrect scan planes. The ratio of correct to incorrect scan planes as rated by the expert is 49:45. This baseline rating is treated as the ground truth while performing quantitative evaluation. Our goal is to develop an algorithm that can produce the scan plane classification results as similar to the domain expert as possible.

In our template-based approach, we utilize 51 ultrasound images of correct scan planes to learn the AAM model. Also, 112 images are used to train the LDA-based score function. Note that all of these images are not part of the 94 test images mentioned earlier. Figure 5 shows the AAM fitting results and the LDA scores of 4 images in the test set. It can be observed that the score is a good indication of how likely the image is a correct scan plane. Figure 6 shows the Receiver operating characteristic (ROC) curve of the test on the entire test set. The Equal Error Rate (EER) of our approach is around $28 \%$ on this data set. In terms of speed, both the AAM-based structure fitting and score function calculation can run in real-time (30+ frames per second) on a conventional PC.
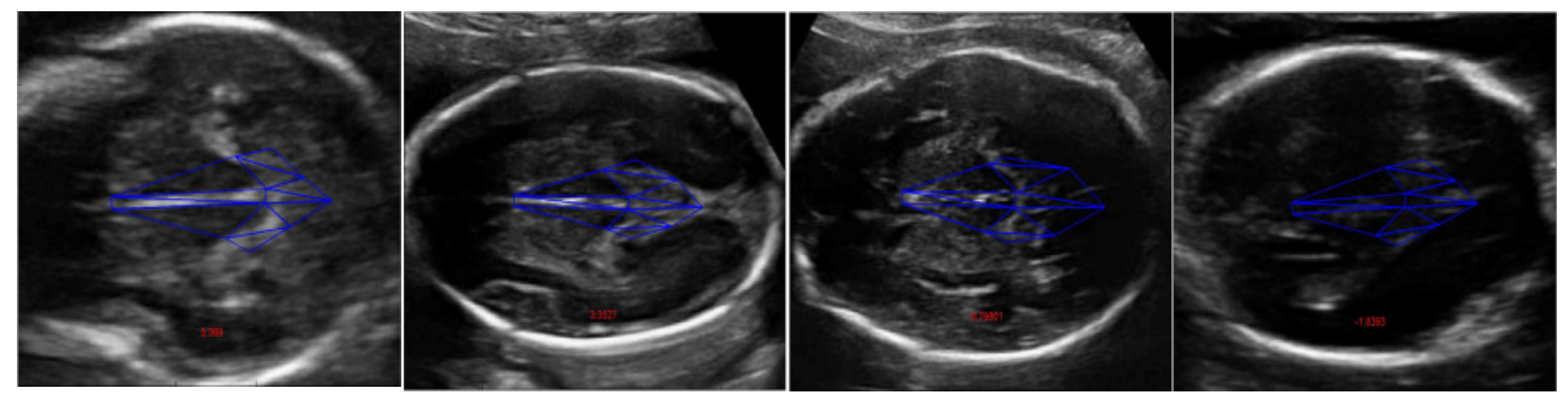

Figure 4. Example scan planes with decreasing scores 5.4, 3.4, 0.8 and -1.8 (from left to right). The classification threshold is 0.6. Notice that imperfections of the head segmentation and hence the rectification are well handled by our structure fitting algorithm. 


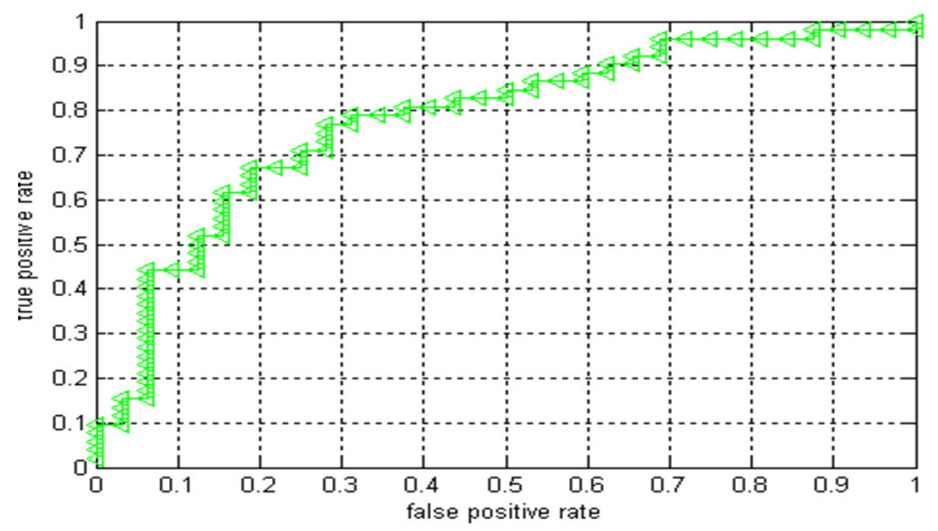

Figure 5. ROC curve of our approach on the test set.

\section{NOVEL OR BREAKTHROUGH WORK}

Within the community of expert sonographers, scan plane identification has been traditionally considered as a problem which eventually becomes simpler with years of practice. In this work, we have developed automated detection and recognition algorithms to simplify clinical workflow that could help in reducing the time of acquisition of scan planes for non-expert users of ultrasound. We propose a novel idea to generate a score for the acceptability of the current frame, which is visible on the console, to make a biometry measurement. ${ }^{13} \mathrm{We}$ believe that this work can be an important pre-curser to many ultrasound measurements which depend on a stringent definition of a 2D scan plane. Unlike the work of Jackson and Hossack ${ }^{14}$ that utilizes the position of the probe and registers it with body coordinates to arrive at the acceptability of the image, our method relies

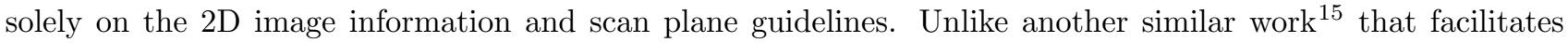
comparison of images across multiple scans by ensuring fixed parameters for all scans, our scoring implicitly captures scanning presets for the anatomy/scan plane of interest through the model learning process. Since we calculate a single normalized score for a standalone image, we could easily apply comparative scoring for a cine loop. In our implementation, the single score is determined by correlating clinical input to real measurable quantity and then normalizing against the variability present in the data. Unlike the work of Stre et al. ${ }^{16}$ that uses a previously acquired reference loop to guide and help acquisition of a specific cut-plane that could be useful in specific applications like needle or catheter guidance, our main goal is to simplify acquisition of scan planes to facilitate measurements. Consequently, we do not require a carefully acquired reference scan. However, our approach enables the user to collect a long video by scanning the entire belly of the patient, and then our algorithm can generate scores for the frames which are pertinent for a particular biometry measurement.

\section{CONCLUSIONS}

In this work we present an automatic method to rate the quality of the fetal head scan plane to enable subsequent biometry measurements. The learning-based AAM method is used to seek the specific structure unique to the correct scan planes. We have demonstrated the performance of our method on a labeled set of scan images. Our future work will focus on combining the proposed method with the feature-based method ${ }^{13}$ to simultaneously leverage their overlap and complementary attributes.

\section{ACKNOWLEDGMENTS}

The authors would like to thank Dr. Madhumita Gupta and Dr. Uday Patil for providing clinical insights and ground truth. 


\section{REFERENCES}

[1] Kimberly, H. H., Murray, A., Mennicke, M., Liteplo, A., Lew, J., Bohan, J. S., Tyer-Viola, L., Ahn, R., Burke, T., and Noble, V. E., "Focused maternal ultrasound by midwives in rural zambia," Ultrasound in Med. \& Biol 36(8), 1267-1272 (2010).

[2] Cootes, T., Edwards, G., and Taylor, C., "Active appearance models," IEEE Trans. on Pattern Analysis and Machine Intelligence 23, 681-685 (June 2001).

[3] Liu, X., Tu, P., and Wheeler, F., "Face model fitting on low resolution images," in [Proc. of the British Machine Vision Conference (BMVC)], 3, 1079-1088 (Sept. 2006).

[4] Liu, X., Wheeler, F., and Tu, P., "Improved face model fitting on video sequences," in [Proc. of the British Machine Vision Conference (BMVC)], (Sept. 2007).

[5] Liu, X., "Video-based face model fitting using adaptive active appearance model," J. Image and Vision Computing 28, 1162-1172 (July 2010).

[6] Liu, X., "Generic face alignment using boosted appearance model," in [Proc. of the IEEE Conf. on Computer Vision and Pattern Recognition (CVPR)], (June 2007).

[7] Liu, X., "Discriminative face alignment," IEEE Trans. on Pattern Analysis and Machine Intelligence 31, 1941-1954 (November 2009).

[8] Wu, H., Liu, X., and Doretto, G., "Face alignment via boosted ranking models," in [Proc. of the IEEE Conf. on Computer Vision and Pattern Recognition (CVPR)], (June 2008).

[9] Liu, X., "Optimal gradient pursuit for face alignment," in [Proc. of Int. Conf. on Automatic Face and Gesture Recognition (FG)], (Mar. 2011).

[10] Banerjee, J. and Krishnan, K. B., "Model-based detection of acoustically dense objects in ultrasound," in [Proc. of the International Conference on Pattern Recognition (ICPR)], 4166-4169 (2010).

[11] Baker, S. and Matthews, I., "Lucas-Kanade 20 years on: A unifying framework," Int. J. Computer Vision 56, 221-255 (March 2004).

[12] Martinez, A. M. and Kak, A. C., "PCA versus LDA," IEEE Trans. on Pattern Analysis and Machine Intelligence 23, 228-233 (2001).

[13] Annangi, P., Krishnan, K., Banerjee, J., Gupta, M., and Patil, U., "Automatic detection and estimation of parietal diameter from fetal ultrasonography," in [SPIE Medical Imaging], 7968 (2010).

[14] Jackson, J. I. and Hossack, J. A., "Medical diagnostic ultrasound system and method for scanning plane orientation," US Patent Issued number 0065265 (April 2003).

[15] Lundberg, V., Steen, E. N., Maehle, J., and Stavo, A., "Methods and apparatus for defining a protocol for ultrasound imaging," US Patent Issued number 0281444 (December 2005).

[16] Stre, D., Stav, A., and Lundberg, V., "Ultrasound image acquisition with synchronized reference image," US Patent Issued number 6488629 (December 2002). 